Konstantinos Poularakis, George Iosifidis, Georgios Smaragdakis, Leandros Tassiulas

\title{
One Step at a Time: Optimizing SDN Upgrades in ISP Networks
}

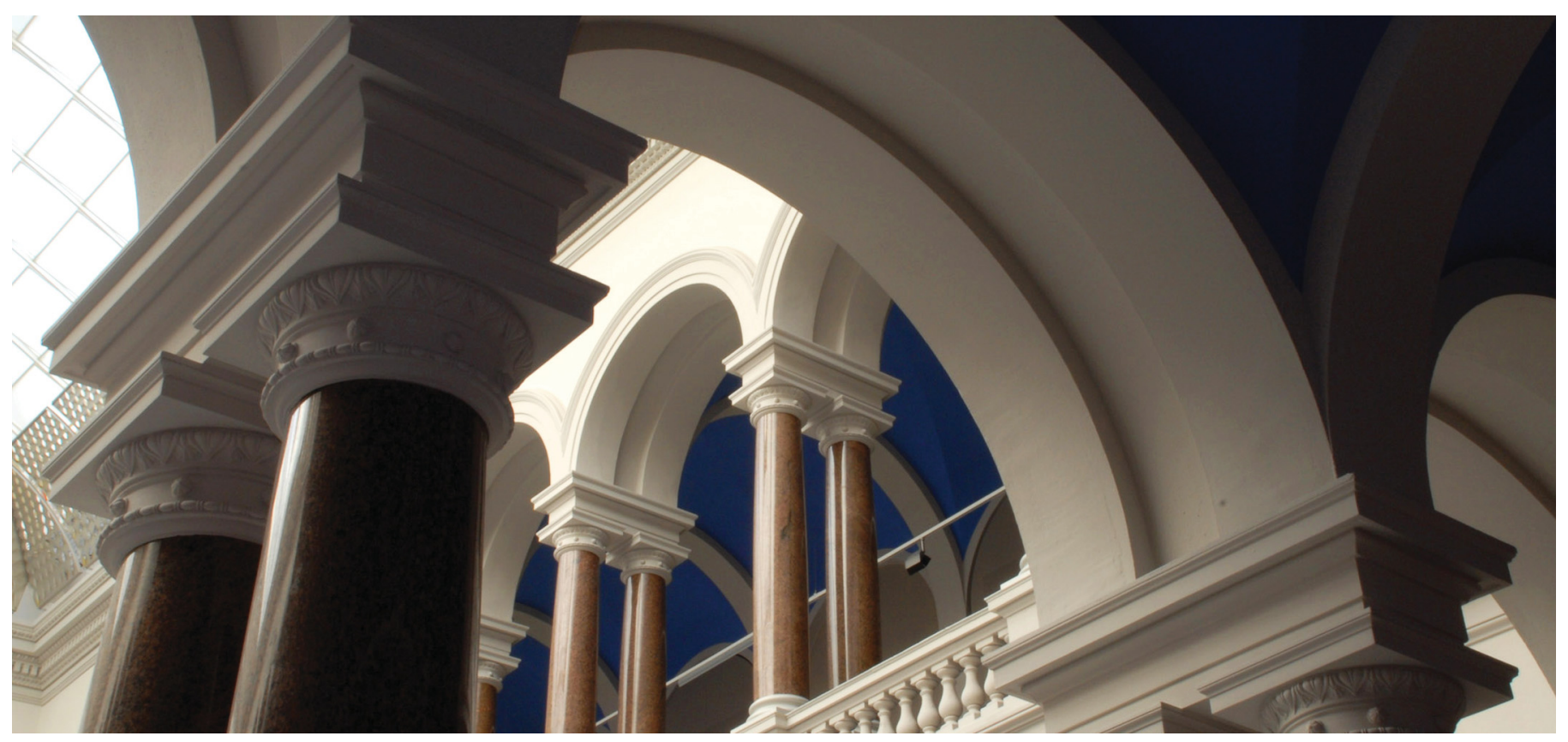

Poularakis, K., Iosifidis, G., Smaragdakis, G., \& Tassiulas, L. (2017). One step at a time: Optimizing SDN upgrades in ISP networks. IEEE INFOCOM 2017 - IEEE Conference on Computer Communications. https://doi.org/10.1109/infocom.2017.8057136 


\title{
One Step at a Time: Optimizing SDN Upgrades in ISP Networks
}

\author{
Konstantinos Poularakis ${ }^{1}$, George Iosifidis ${ }^{1,2}$, Georgios Smaragdakis ${ }^{3}$, and Leandros Tassiulas ${ }^{1}$ \\ ${ }^{1}$ Department of Electrical Engineering and Institute for Network Science, Yale University, USA \\ ${ }^{2}$ School of Computer Science and Statistics, and CONNECT, Trinity College Dublin, Ireland \\ ${ }^{3}$ MIT and TU Berlin
}

\begin{abstract}
Nowadays, there is a fast-paced shift from legacy telecommunication systems to novel Software Defined Network (SDN) architectures that can support on-the-fly network reconfiguration, therefore, empowering advanced traffic engineering mechanisms. Despite this momentum, migration to SDN cannot be realized at once especially in high-end cost networks of Internet Service Providers (ISPs). It is expected that ISPs will gradually upgrade their networks to SDN over a period that spans several years. In this paper, we study the SDN upgrading problem in an ISP network: which nodes to upgrade and when. We consider a general model that captures different migration costs and network topologies, and two plausible ISP objectives; first, the maximization of the traffic that traverses at least one SDN node, and second, the maximization of the number of dynamically selectable routing paths enabled by SDN nodes. We leverage the theory of submodular and supermodular functions to devise algorithms with provable approximation ratios for each objective. Using real-world network topologies and traffic matrices, we evaluate the performance of our algorithms and show up to $54 \%$ gains over state-of-the-art methods. Moreover, we describe the interplay between the two objectives; maximizing one may cause a factor of 2 loss to the other.
\end{abstract}

\section{INTRODUCTION}

Motivation. Software Defined Networking (SDN) [1] enables unprecedented network management flexibility through the separation of the network control and data planes, and the centralization of the former in designated network entities, referred to as controllers. A controller maintains a global view of the network state, including network topology, traffic load and link failures, and can leverage this information to dynamically select the routing paths for each network flow. This approach departs significantly from traditional IP protocols, like OSPF, that are destination-based and route traffic along shortest paths using static link weight metrics. SDN, therefore, empowers advanced Traffic Engineering (TE) mechanisms that can respond on-the-fly to network changes, and support fine-grained routing decisions per flow. This makes SDN a particularly attractive technology.

However, as it happens with most novel network protocols and architectures [2], migration to SDN cannot be realized at once. This is particularly true for the large and expensive networks of Internet Service Providers (ISPs). Namely, the one-step SDN upgrade of entire ISP networks is practically impossible since it poses an enormous operational burden, and also raises performance and security risks [3]. On top of that, such upgrades require huge capital expenditures since network

K. Poularakis and L. Tassiulas were supported by the National Science Foundation under Grant CNS 1527090. G. Smaragdakis was supported by the ERC Starting Grant ResolutioNet (ERC-StG-679158).

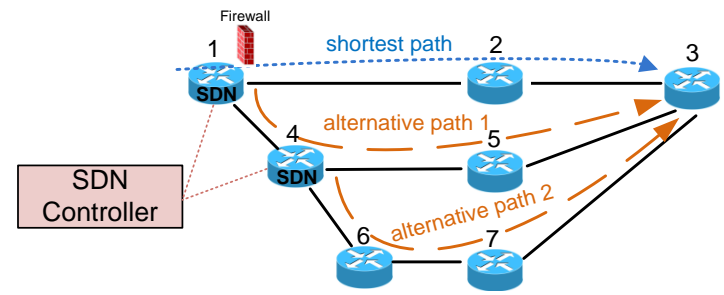

Fig. 1. A network that is partially upgraded to SDN. The two SDN nodes can act as firewalls or dynamically control the routing path.

components (e.g., backbone routers) are very expensive. Besides, upgrading newly installed legacy routers is economically prohibitive ${ }^{1}$. Given the above, it is expected that ISPs will opt to migrate to SDN incrementally, i.e., by gradually upgrading their network nodes over a period that spans several years. In these incremental SDN deployments, the controllers will manage only the SDN-enabled nodes, while the remaining legacy network will still use OSPF-like routing protocols.

Even in such hybrid SDN networks, the ISPs can accrue important benefits. Namely, for the traffic that crosses at least one SDN node, it is possible to apply various sophisticated policies such as access control, firewall actions, and other middlebox-supported in-network services [5]. Moreover, using the SDN nodes it is possible to dynamically control the routing path of the flows by overriding the underlying legacy OSPF protocol [6]. In line with prior works, we will use the term programmable traffic to differentiate the traffic that traverses at least one SDN node from that not traversing any SDN nodes. Both in-network services and the availability of alternative routing paths (that can be dynamically selected) are extremely useful for ISPs. Besides, if the flow crosses more than one SDN nodes, the ISP has even more dynamic routing options and hence can further increase the TE flexibility of its network.

Let us show the potential of this approach with a simple example. Consider the hybrid SDN toy-network shown in Figure 1 that routes a flow from source node 1 to destination node 3. Here, only two of the seven nodes are upgraded with SDN capabilities (nodes 1 and 4). Using OSPF, the flow is always routed along the shortest path. However, node 1 can dynamically decide to drop (instead of forwarding) the packets, acting, e.g., as a firewall. It can also override the OSPF shortest path by routing the packets through node 4 . The packets will then follow the alternative path 1 which

\footnotetext{
${ }^{1}$ A typical router replacement window is 3 to 5 years; more importantly a network's routers have out-of-phase cycles, i.e., need to be replaced in different times, e.g., see Lifecycle Financing from Cisco Capital. Also, router costs vary significantly from few tens of thousands to more than $\$ 100 K$ [4].
} 
is the OSPF shortest path connecting node 4 with 3 . Such flow rerouting is important when a link of the shortest path fails or becomes temporarily congested. Since node 4 is also upgraded to SDN, it can similarly defer packets towards alternative path 2. In other words, as the number of SDNenabled nodes increases, the set of alternative paths increases as well. Hence, there exist more degrees of freedom (or, flexibility) in performing dynamic TE.

To gain the maximum benefits, it is important to identify which SDN upgrade schedule is suitable for a given network. Namely, every ISP needs to carefully select which nodes to upgrade, and when exactly to do so. Especially this latter aspect of timing has many implications. First, like every new technology, the initial high cost of SDN decreases with high pace over time [7]. Hence the ISPs face a dilemma of early upgrade that will allow them to reap the new technology benefits immediately and a slow upgrade that will reduce their capital expenditures. More practical, the ISPs need to decide how many nodes to upgrade in each period, which for ISPs usually amounts to 6-12 month intervals. Second, the routers are highly heterogeneous since they serve a different amount of traffic and have a different remaining lifetime, and this further perplexes these decisions.

In summary, every ISP has to address the following two questions: (i) How many nodes to upgrade in each period? Should it upgrade all nodes as early as possible or wait for the prices to fall?, and (ii) After deciding the number of nodes to be upgraded, which specific nodes to select? The ISP's goal might be to maximize the volume of programmable traffic or the TE flexibility by increasing the dynamically selectable alternative paths, based on the ISP's priorities and preferences. Despite the very important recent prior works on hybrid SDN networks, e.g., see [6], [8] and Section VII for a detailed overview, we currently lack a systematic understanding regarding the above issues. Therefore, our goal in this work is to investigate policies for SDN upgrade scheduling in large (and expensive) operational ISP networks, and focus particularly on the impact of time-dimension and the interplay between traffic programmability and TE flexibility benefits.

Methodology and Contributions. We develop a methodology to address the above two questions posed by ISPs regarding SDN migration. We introduce a model of SDN upgrades general enough to capture different migration costs, as well as ISP topologies and traffic demands. We then utilize this model to derive the optimal scheduling for router upgrades in the ISP network over a period that may span several years. We consider two ISP objectives. First, we target the maximization of the programmable traffic, i.e., the traffic that traverses at least one SDN node $(O b j 1)$. This upgrading policy, if designed properly, can have significant benefits [6], [8], [9], since it allows an ISP to control how the traffic flows in its own network. The second objective $(O b j 2)$ aims to maximize the TE flexibility. This objective is achieved by increasing the number of alternative paths through the SDN upgrades. For each one of the two objectives we formulate a rigorous optimization problem and devise the desirable SDN upgrading policy (or, schedule): which nodes to upgrade and when.

In both cases, finding the upgrading policy requires the solution of challenging combinatorial optimization problems. Namely, we show that for Obj1 this problem is NP-Hard even to approximate to any factor better than $1-1 / e$. For the special case in which all the node upgrades take place at the same time period, we show that a modified version of a classic greedy algorithm, which enumerates all possible triplets of nodes as candidate solutions, achieves the best possible approximation ratio. We also show a simple way to extend this algorithm for the general case where the node upgrades can take place at different time periods. A second class of more sophisticated algorithms with improved approximation ratios is presented by expressing Obj1 as the maximization of a submodular set function [10], i.e., a function that satisfies the diminishing returns property. Then, we study Obj2 (maximizing TE flexibility). This is a more complex problem which can be expressed as the maximization of a function with bounded supermodular degree [11]. Using this result, we present another greedy-based algorithm that approximately solves this problem. We evaluate the performance of the proposed algorithms using two datasets of real network topologies and traffic matrices [12], [13]. The results clearly differentiate situations in which upgrades should be spread over many instead of one step.

The contributions of this work are summarized as follows:

- SDN Upgrading Problem. We introduce the problem of gradually (and partially) upgrading an ISP network to SDN, using general models of costs and different objectives. The upgrades can take place at different time periods, introducing different costs at each period due to technology maturity, the different life-cycle of the network equipment and other practical limitations.

- Maximizing Programmable Traffic (Obj1). For the programmable traffic maximization objective, we show that the SDN upgrading problem is NP-Hard to approximate to any factor better than $1-1 / e$. Then, we present a simple algorithm matching this factor for the special case of one time period, and show how it can be extended for the general case. We also present additional more sophisticated approximation algorithms using the theory of submodular functions.

- Maximizing TE Flexibility (Obj2). For the objective of maximizing TE flexibility through the availability of SDN-enabled routing paths, we show that the optimization problem is more complex. We present an approximation algorithm by expressing it as the maximization of a function with bounded supermodular degree.

- Dataset-driven Evaluation. We evaluate the proposed algorithms using real-world network topologies and traffic matrices. We find that our approach can increase by $54 \%$ the amount of programmable traffic compared to two state-of-the-art methods in practical scenarios. We also find that by optimizing Obj1, benefits are also realized for Obj2 (and vise versa) and we explore the interplay between the two objectives.

The rest of the paper is organized as follows. Section II describes the system model and formalizes the SDN upgrading problem for Obj1. In Sections III and IV, we present theoretical results about the computational complexity of this problem and approximation algorithms. Section V considers Obj2 and 
presents an approximation algorithm. Section VI presents the dataset-driven evaluation of our proposed algorithms, while Section VII reviews our contribution compared to related works. We conclude our work in Section VIII.

\section{Model ANd Problem Formulation}

We adopt a general model representing a large ISP backbone network with a set $\mathcal{N}$ of $N$ nodes (e.g., routers). The network traffic consists of a set $\mathcal{F}$ of $F$ origin-destination flows. With traditional IP routing protocols, like OSPF, each flow $f$ follows the shortest path to the destination. We denote with $\mathcal{N}_{f} \subseteq \mathcal{N}$ the set containing the nodes along this path. The ISP may decide to upgrade some of the nodes to SDN, and makes these decisions along a time interval of $t=1, \ldots, T$ time periods, $t \in \mathcal{T}$. Typically, such decisions are made in an annual or semi-annual fashion, and by accommodating the lifetime of this type of equipment (3-5 years). Thus, a usual value can be $T=5$ or 10 time periods. An example of incremental SDN upgrades is depicted in Figure 2.

Moreover, the global Internet traffic increases with time, having an expected annual growth rate of 22 percent from 2015 to 2020 [14]. To capture these dynamics, we denote with $\lambda_{t f}$ (bps) the average rate of flow $f$ at period $t$, where $\lambda_{t f} \geq \lambda_{t^{\prime} f}, \forall t>t^{\prime}$. Although traffic variations may appear also within the same period, it is expected that in backbone networks with high aggregation of flows the traffic will not to be very volatile. Upgrading a node to SDN requires capital expenditures, e.g., for buying a new SDN-enabled device, installation costs, etc. These costs typically differ across nodes. For example, upgrades to edge nodes are typically less expensive than core network nodes, while it is definitely more costefficient to upgrade a node at the end of its lifetime (rather than a newly installed one). Besides, the costs are likely to drop over time as the SDN technology matures. We denote with $b_{t n}$ (in USD) the cost for upgrading node $n$ at period $t$, where it may be $b_{t n} \neq b_{t n^{\prime}}, n \neq n^{\prime}$, and $b_{t n} \leq b_{t^{\prime} n}, t \geq t^{\prime}$.

We introduce the optimization variable $x_{t n} \in\{0,1\}$ that indicates whether node $n \in \mathcal{N}$ will be upgraded to SDN at time period $t$ or not. These variables constitute the upgrading policy of the ISP:

$$
\boldsymbol{x}=\left(x_{t n} \in\{0,1\}: t \in \mathcal{T} n \in \mathcal{N}\right) .
$$

We consider the case that the ISP has an available monetary budget $B$ (in USD) that can invest in SDN upgrades. The ISP may opt to either invest this capital at once, or spread the budget over different time periods. In any case, the SDN upgrading policy must satisfy the following constraint:

$$
\sum_{t \in \mathcal{T}} \sum_{n \in \mathcal{N}} x_{t n} b_{t n} \leq B
$$

Clearly, each node can be upgraded to SDN at most once:

$$
\sum_{t \in \mathcal{T}} x_{t n} \leq 1, \forall n \in \mathcal{N} \text {. }
$$

Since the network is upgraded incrementally, some flows may still traverse only legacy nodes, or they may traverse a mixture of upgraded and legacy nodes. A flow that traverses at least one SDN node, can be used to realize a programmatic

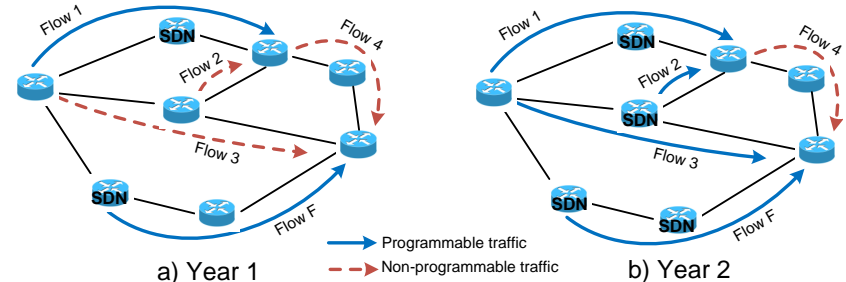

Fig. 2. An example of incremental SDN upgrades for $T=2$ years. Two nodes are upgraded each year, increasing the amount of programmable traffic.

interface, e.g., for implementing a firewall or routing traffic to alternative paths (as we depicted in Figure 1). The volume of programmable traffic can be expressed as follows:

$$
J_{1}(\boldsymbol{x})=\sum_{t \in \mathcal{T}} \sum_{f \in \mathcal{F}} \lambda_{t f} 1_{\left\{\sum_{n \in \mathcal{N}_{f}} \sum_{t^{\prime} \leq t} x_{t^{\prime} n} \geq 1\right\}}
$$

Here, $1_{\{.\}}$is the indicator function; it is equal to one if the condition in the subscript is true, otherwise zero.

The first natural objective of the ISP is to find the upgrading policy that maximizes the volume of programmable traffic (PTM problem):

$$
\begin{array}{ccc}
\text { Obj1: } & \max _{\boldsymbol{x}} & J_{1}(\boldsymbol{x}) \\
& \text { s.t. } & \text { constraints }:(2),(3) \\
& & x_{t n} \in\{0,1\}, \forall t \in \mathcal{T}, n \in \mathcal{N} .
\end{array}
$$

This is a challenging combinatorial optimization problem. For example, in a network of $N=100$ nodes and $T=10$ time periods there will be $2^{N T}=2^{1000}$ possible solutions, and therefore brute force algorithms that exhaustively search the solution space will require prohibitively large running time.

The second plausible ISP objective that we examine (Obj2), is the maximization of the TE flexibility. The latter is directly proportional to the number of alternative routing paths that are dynamically selectable through the SDN upgraded nodes. We present the formulation of this objective in Section V.

\section{Complexity Analysis of the PTM Problem}

In this section, we investigate thoroughly the complexity of the PTM problem, and we propose a simple algorithm with a provable approximation ratio. We begin with the special case of $T=1$ period and then extend our results for any $T$.

Theorem 1: For the special case of $T=1$ time period, the PTM problem is NP-Hard, but there exists a polynomial-time $(1-1 / e)$-approximation algorithm.

In order to prove Theorem 1 we will consider a variant of the coverage problem known as Budgeted Maximum Coverage Problem (BMCP) [15]. The latter can be defined as follows.

$B M C P$ : Given a set of elements $\mathcal{E}=\left\{E_{1}, E_{2}, \ldots, E_{l}\right\}$ with associated weights $\left\{w_{i}\right\}_{i=1}^{l}$ and a collection of sets $\mathcal{S}=\left\{S_{1}, S_{2}, \ldots, S_{m}\right\}$ defined over $\mathcal{E}$ with associated costs $\left\{c_{i}\right\}_{i=1}^{m}$, the goal is to find a collection of sets $\mathcal{S}^{\prime} \subseteq \mathcal{S}$, such that the total cost of sets in $\mathcal{S}^{\prime}$ does not exceed budget $C$ and the total weight of elements covered by $\mathcal{S}^{\prime}$ is maximized.

Then, we will prove the following Lemma.

Lemma 1: PTM for $T=1$ and BMCP are equivalent problems. 
Proof: Given an arbitrary instance of the BMCP, we construct a specific instance of PTM problem for a single period $t$ as follows. We create a separate node for each set, i.e., $\mathcal{N}=\{1,2, \ldots,|\mathcal{S}|\}$. The upgrading costs of the nodes match the costs of the sets, i.e., $b_{t n}=c_{S(n)}$ where the set $S(n)$ corresponds to node $n$. The upgrading budget $B$ matches the budget $C$ in the BMCP instance. We also create a separate flow for each element, i.e., $\mathcal{F}=\{1,2, \ldots,|\mathcal{E}|\}$. The flow rates match the weights of the elements, i.e., $\lambda_{t f}=w_{E(f)}$ where the element $E(f)$ corresponds to flow $f$.

If there exists a set of nodes which upgrading them to SDN makes $Q$ amount of traffic to traverse at least one SDN node (programmable traffic), then there will also be a collection of sets that cover elements with total weight $Q$. The covered elements will correspond to the flows that traverse the SDN nodes. The total cost of the node upgrades will be equal to the total cost of the sets. Hence, the solution to the PTM problem provides a solution to the BMCP with the same value. Conversely, given a solution to the BMCP problem, one can solve the PTM problem by upgrading the nodes corresponding to the sets picked by the BMCP solution. The two solutions will have the same value.

It is known that it is NP-Hard to approximate the BMCP problem to any factor better than $1-1 / e$ [15]. Therefore, Lemma 1 indicates that the same statement holds for the PTM problem. Hence, we have proved the first part of Theorem 1. To prove the second part, we leverage an algorithm in literature that is used for solving the BMCP. Specifically, the work in [15] has shown that a modification of the well-known greedy algorithm yields an $(1-1 / e)$-approximate solution to BMCP (matching the best possible factor). When applied to the PTM problem for $T=1$, this algorithm enumerates all subsets of nodes with cardinality 3 , that have total cost at most $B$. It completes each subset to a candidate solution in a greedy fashion. Specifically, it iteratively upgrades the node with the highest ratio of the traffic that becomes programmable over upgrading cost. Another set of candidate solutions consists of all subsets of nodes with cardinality less than 3 , which have total cost at most $B$. The algorithm will output the candidate solution that achieves the highest programmable traffic volume. The pseudocode of the algorithm is presented in Algorithm 1, and described in the sequel.

Here, $C S_{1}, C S_{2} \subseteq \mathcal{N}$ are the two candidate solutions found by the algorithm. $C S_{1}$ is initialized to the empty set (line 1). $\mathcal{U}$ denotes the set of nodes that are not included in the current candidate solution. $\Delta J_{1}\left(\mathcal{N}^{\prime}, n\right)$ indicates the additional traffic that becomes programmable when node $n$ is upgraded, given that the nodes in subset $\mathcal{N}^{\prime}$ are already upgraded. The term $\boldsymbol{x}\left(C S_{1}\right)$ indicates the vector $\boldsymbol{x}$ for which $x_{n}=1$ iff $n \in C S_{1}$. Similarly, for $\boldsymbol{x}\left(C S_{2}\right)$. For each possible triplet of upgraded nodes, the algorithm iteratively upgrades the node with the highest ratio of traffic that becomes programmable over upgrading cost (line 4). Nodes will be skipped if their upgrade violates the budget constraint (line 5). At the end of the loop (line 8), $C S_{1}$ will be the solution with the best value. $C S_{2}$ will be the best solution of cardinality less than 3. The algorithm will compare $C S_{1}$ and $C S_{2}$ and pick the solution with the best value (lines 9-10).

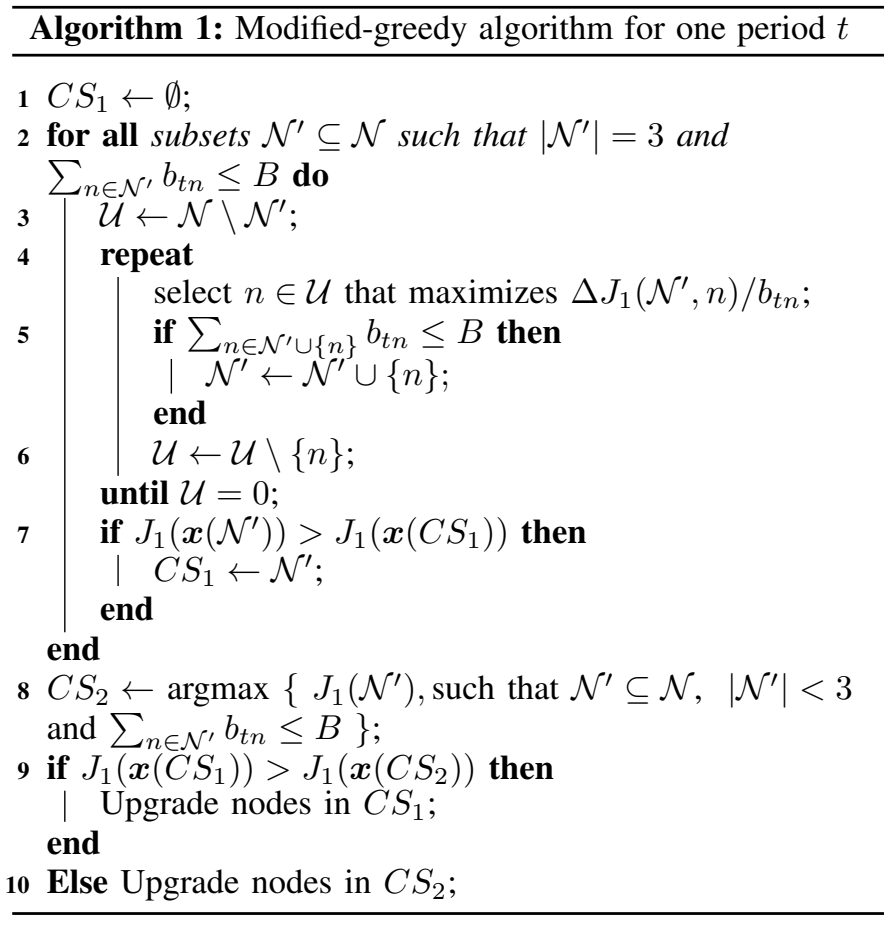

With the Modified-greedy algorithm, we have proved the second part of Theorem 1. Nevertheless, this algorithm works only for a single time period $(T=1)$. The rest of this section extends the results for the general case with many time periods.

Theorem 2: For the general case, there exists an $O((1-$ $1 / e) / \log (T))$-approximation algorithm to PTM problem.

We will prove this theorem by extending any approximation algorithm that works for one period to many periods. Formally, we prove the following lemma.

Lemma 2: We can extend any a-approximation algorithm for the $T=1$ case of the PTM problem, to obtain an $O(a / \log (T))$-approximation for the general $T>1$ case.

Proof: We denote with $A L G$ an $a$-approximation algorithm of the PTM problem for the $T=1$ case. We also consider the general case of the PTM problem (with $T>1$ ), but neglect constraints (2), (3) and assume that the available budget in each one of the time periods is $B$ (hence the total budget is $T \cdot B$ ). In this relaxed version, the PTM problem can be decomposed into $T$ independent subproblems, one for each time period. We can run $A L G$ for each subproblem $t \in\{1,2, \ldots, T\}$ to obtain a solution $A L G_{t}$ with value $v\left(A L G_{t}\right) \geq a \cdot v\left(O P T_{t}\right)$, where $O P T_{t}$ is the respective optimal solution. Then, we transform each solution $A L G_{t}$ to a feasible solution $H_{t}$ to the original PTM problem as follows. In the $H_{t}$ solution, we perform no SDN upgrades during the first $t-1$ periods, or after the $t^{t h}$ period. All the upgrades take place at the $t^{t h}$ period based on the $A L G_{t}$ solution. Hence, the programmable traffic during the first $t-1$ periods will be zero. Since the traffic rate increases with time, the programmable traffic will be at least $(T-t+1) \cdot v\left(A L G_{t}\right)$ during the rest $T-t+1$ periods. Out of these $T$ solutions $\left(H_{1}\right.$ to $\left.H_{T}\right)$, we return the one of maximum value, denoted with $H^{*}$. Then, for 
all $t$ it should hold that:

$v\left(H^{*}\right) \geq(T-t+1) \cdot v\left(A L G_{t}\right) \geq(T-t+1) \cdot a \cdot v\left(O P T_{t}\right)$,

and therefore,

$$
v\left(O P T_{t}\right) \leq \frac{1}{a \cdot(T-t+1)} \cdot v\left(H^{*}\right) .
$$

If $O P T^{*}$ is the optimal solution to the PTM problem, then

$$
\begin{aligned}
v\left(O P T^{*}\right) & \leq \sum_{t=1}^{T} v\left(O P T_{t}\right) \leq v\left(H^{*}\right) \cdot \sum_{t=1}^{T} \frac{1}{a \cdot(T-t+1)} \\
& =v\left(H^{*}\right) \cdot O\left(\frac{\log (T)}{a}\right)
\end{aligned}
$$

where the first inequality is because the solutions $O P T_{t}, \forall t$ are computed neglecting the constraints (2) and (3). The second inequality is because of (6). The last equation is based on the definition of the $T^{t h}$ harmonic number.

Theorems 1 and 2 analyze the complexity of the PTM problem and provide a first approximate solution. Although it is quite simple to implement, Modified-greedy provides an approximation ratio that worsens as the number of periods $T$ increases. To fill this gap, we introduce in the next section a class of (more sophisticated) algorithms with approximation ratios that are independent of $T$, and therefore are suitable for upgrade schedules that extend over long time windows.

\section{Tight Approximations For the PTM PROBlem}

In this section, we present tight approximation algorithms for the PTM problem by expressing the problem as the maximization of a submodular function. We begin by introducing the definition of submodular functions.

Definition 1: Given a finite set of elements $\mathcal{G}$ (referred to as ground set) a function $H: 2^{\mathcal{G}} \rightarrow \mathbb{R}$ is submodular if for any sets $\mathcal{A} \subseteq \mathcal{B} \subseteq \mathcal{G}$ and every element $g \in \mathcal{G} \backslash \mathcal{B}$, it holds:

$$
H(\mathcal{A} \cup\{g\})-H(\mathcal{A}) \geq H(\mathcal{B} \cup\{g\})-H(\mathcal{B}),
$$

i.e., the marginal value of the function when adding a new element in a set decreases as this set expands.

Let us denote the upgrade of node $n$ at time period $t$ by an element $g_{t n}$ and define the ground set $\mathcal{G}$ consisting of all elements as:

$$
\mathcal{G}=\left(g_{t n}: t \in \mathcal{T}, n \in \mathcal{N}\right) .
$$

Then, every possible SDN upgrading policy can be expressed by a subset $\mathcal{X} \subseteq \mathcal{G}$, where the elements in $\mathcal{X}$ correspond to the time periods and nodes that upgrades took place. Based on the above, we can write Obj 1 as a function of the set $\mathcal{X}$ :

$$
H(\mathcal{X})=\sum_{t \in \mathcal{T}} \sum_{f \in \mathcal{F}} \lambda_{t f} 1_{\left.\left\{\bigcup_{t^{\prime} \leq t, n \in \mathcal{N}_{f}} g_{t^{\prime} n}\right\} \cap \mathcal{X} \neq \emptyset\right\}} \cdot
$$

Then, we prove the following lemma:

Lemma 3: The function $H(\mathcal{X})$ is monotone, increasing and submodular.

Proof: Monotonicity is obvious since any new upgrade of a node cannot decrease the value of the objective function. In order to show submodularity, we observe that since the sum of submodular functions is also submodular it suffices to show

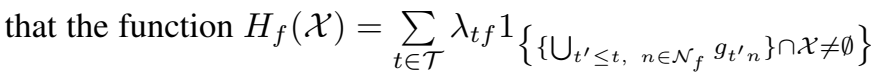

for a given flow $f$ is submodular. To this end, we consider two SDN upgrading policies $\mathcal{A} \subseteq \mathcal{G}$ and $\mathcal{B} \subseteq \mathcal{G}$, where $\mathcal{A} \subseteq \mathcal{B}$. We also consider an element $g_{t n} \in \mathcal{G} \backslash \mathcal{B}$ to be added to both sets. This corresponds to upgrading node $n$ at period $t$, where this upgrade was not taken place neither in set $\mathcal{A}$ nor $\mathcal{B}$.

The marginal value for adding $g_{t n}$ to $\mathcal{A}$ will be zero if there exists another element $g_{t^{\prime} n^{\prime}} \in \mathcal{A}$ such that $t^{\prime} \leq t$ and $n^{\prime} \in \mathcal{N}_{f}$. This is because the flow $f$ is already programmable according to policy $\mathcal{A}$. Else if there exists an element $g_{t^{\prime \prime} n^{\prime}} \in$ $\mathcal{A}$ such that $t^{\prime \prime}>t$ and $n^{\prime} \in \mathcal{N}_{f}$, then the marginal value will be $\sum_{i=t}^{t^{\prime \prime}-1} \lambda_{i f}$. This is because the flow $f$ now becomes programmable at time $t$ instead of $t^{\prime \prime}$. Otherwise, the marginal value will be $\sum_{i=t}^{T} \lambda_{i f}$.

We now consider the marginal value for adding the element $g_{t n}$ to the set $\mathcal{B}$. We distinguish the following three cases:

(i) If there exists an element $g_{t^{\prime} n^{\prime}} \in \mathcal{A}$ such that $t^{\prime} \leq t$ and $n^{\prime} \in \mathcal{N}_{f}$ then this element will also belong to $\mathcal{B}$. Hence, the marginal value will be zero for both $\mathcal{A}$ and $\mathcal{B}$.

(ii) If there exists an element $g_{t^{\prime \prime} n^{\prime}} \in \mathcal{A}$ such that $t^{\prime \prime}>t$ and $n^{\prime} \in \mathcal{N}_{f}$ then this element will also belong to $\mathcal{B}$. We distinguish the following two subcases. (ii.a) If there exists an element $g_{t^{\prime \prime \prime} n^{\prime}} \in \mathcal{B} \backslash \mathcal{A}$ such that $t^{\prime \prime \prime}<t^{\prime \prime}$ the marginal value for $\mathcal{B}$ will be $\sum_{i=t}^{t^{\prime \prime \prime}-1} \lambda_{i f}<\sum_{i=t}^{t^{\prime \prime}-1} \lambda_{i f}$. (ii.b) Otherwise, the marginal value for $\mathcal{B}$ will be equal to that for $\mathcal{A}\left(\sum_{i=t}^{t^{\prime \prime}-1} \lambda_{i f}\right)$. (iii) In any other case, the marginal value for the set $\mathcal{A}$ will be $\sum_{i=t}^{T} \lambda_{i f}$. But, note that by definition this is the largest possible marginal value for the $\mathcal{B}$ set as well. Hence, in all cases we showed that the marginal value will be lower or equal for the set $\mathcal{B}$ than $\mathcal{A}$.

The ground set can be partitioned into $N$ disjoint sets, $\mathcal{G}_{1}, \mathcal{G}_{2}, \ldots, \mathcal{G}_{N}$, where $\mathcal{G}_{n}=\left\{g_{\text {tn }}: \forall t \in \mathcal{T}\right\}$. Since each node can be upgraded in at most one time period, it should be $\mathcal{X} \in \mathcal{I}_{1}$ where:

$$
\mathcal{I}_{1}=\left\{\mathcal{X} \subseteq \mathcal{G}:\left|\mathcal{X} \cap \mathcal{G}_{n}\right| \leq 1, \forall n \in \mathcal{N}\right\}
$$

Here, the pair $\left(\mathcal{G}, \mathcal{I}_{1}\right)$ forms a partition matroid constraint [10]. Also, due to the budget constraint, it should be $\mathcal{X} \in \mathcal{I}_{2}$ where:

$$
\mathcal{I}_{2}=\left\{\mathcal{X} \subseteq G: \sum_{g_{t n} \in \mathcal{X}} b_{t n} \leq B\right\} .
$$

Here, the pair $\left(\mathcal{G}, \mathcal{I}_{2}\right)$ forms a knapsack constraint.

There exist various approximation algorithms for the maximization of a monotone submodular function subject to a matroid and a knapsack constraint. The algorithm with the best approximation ratio was proposed in [16]. This algorithm uses pipage rounding, a procedure which aims to convert a fractional solution of an optimization problem into an integral one, through a sequence of simple updates. It achieves an $(1-1 / e-\epsilon)$-approximation ratio for any constant $\epsilon>0$. Nevertheless, the value of this algorithm is mostly theoretical, since it relies on the enumeration of $1 / \epsilon^{4}$ elements which can be a quite large number in practice.

A more practical choice is the technique presented in [17]. The idea is to reduce the knapsack constraint into a collection of partition matroids using an enumeration method. Particularly, let us denote with $\left\{u_{1}, u_{2}, \ldots, u_{l}\right\}$ all the different values of the upgrade costs $b_{t n}, \forall t, n$ of the elements in the 
ground set. For example, $l=1$ if all elements have the same cost, but $l=N T$ if each element has a different cost. Then, we can partition the ground set into the sets $\mathcal{G}_{1}^{\prime}, \mathcal{G}_{2}^{\prime}, \ldots, \mathcal{G}_{l}^{\prime}$, where the $i^{\text {th }}$ set contains all elements with cost $u_{i}$. We also define $q_{i}=\left\lfloor B / u_{i}\right\rfloor$. In other words, $q_{i}$ is the maximum number of elements in $\mathcal{G}_{i}^{\prime}$ that can be included in a solution without violating the knapsack constraint. Clearly, there will be at most $m=\prod_{i=1}^{l} q_{i}$ different solutions that satisfy the knapsack constraint, one solution for each combination. For each combination $j \in\{1,2, \ldots, m\}$ we introduce the following partition matroid:

$$
\mathcal{I}_{2}^{j}=\left\{\mathcal{X} \subseteq \mathcal{G}:\left|\mathcal{X} \cap \mathcal{G}_{i}^{\prime}\right| \leq q_{i}^{j}, \forall i \in\{1,2, \ldots, l\}\right\}
$$

where $q_{i}^{j}$ denotes the maximum number of elements in $\mathcal{G}_{i}^{\prime}$ corresponding to the $j^{\text {th }}$ combination.

Clearly, instead of maximizing $H($.$) function with respect$ to the $\mathcal{I}_{1} \cap \mathcal{I}_{2}$ constraint, it is equivalent to maximize $H($. with respect to $\mathcal{I}_{1} \cap \mathcal{I}_{2}^{j}, \forall j \in\{1,2, \ldots, m\}$. The final solution will be simply the best performing of the $m$ solutions found. In other words, with this enumeration method, we replace a knapsack constraint $\left(\mathcal{I}_{2}\right)$ with a collection of matroid constraints $\left(\mathcal{I}_{2}^{j}, j \in\{1,2, \ldots, m\}\right)$. Hence, the PTM problem can be expressed as a collection of $m$ subproblems where in each subproblem a submodular function is maximized subject to $k=2$ partition matroid constraints.

A local search algorithm provides an $(1 /(k+\epsilon))$ approximation for the maximization of a submodular function subject to $k$ partition matroid constraints [10]. This technique takes as input a parameter $\epsilon>0$ and maintains a solution set $\mathcal{X}$ which is always independent in each of the $k$ matroids. Iteratively, the algorithm tries to add at most $2 / \epsilon$ elements and delete at most $2 k / \epsilon$ elements from $\mathcal{X}$. If there is a local move that generates a feasible solution and improves the objective value, the algorithm repeats the local search procedure with that new solution, until no improvement is possible. The procedure is summarized in Algorithm 2.

We emphasize that the enumeration technique that we presented will be very efficient in cases that many nodes have the same upgrade costs ( $l$ is a small number) and the budget is relatively small compared to the upgrading costs $\left(q_{i}\right.$ values are small numbers). Nevertheless, there may be cases in which a large number of matroid constraints need to be enumerated. In order to reduce complexity in these cases, a different enumeration method can be applied. For example, the enumeration method in [16] that approximately reduces every knapsack to a polynomial-time computable collection of matroid constraints can be used. The size of the collection can be tuned depending on how well the knapsack is approximated by the matroid constraints (cf. Lemma 3.3. in [17]).

The pipage rounding and the local search algorithms provide approximation ratios that are independent of the number of periods $T$. Specifically, the following theorem holds:

Theorem 3: There exist an $(1-1 / e-\epsilon)$-approximation algorithm and an $(1 /(2+\epsilon))$-approximation algorithm to the PTM problem for any $\epsilon>0$.

\section{OPtimizing THE TE FLEXIBILITY}

In this section, we study the SDN upgrading problem when the ISP's goal is to maximize the TE flexibility. This

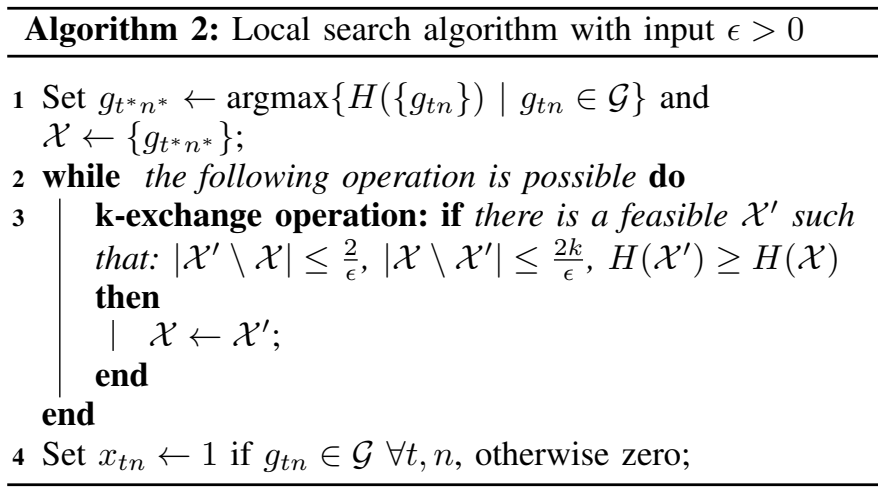

is achieved through the availability of alternative paths that can be dynamically activated in such hybrid SDN networks. Namely, the ISP can use these paths to avoid congestion in cases that certain links are temporarily overloaded or failed. For example, as explained in Figure 1, when node 1 is upgraded the flow can be dynamically routed towards the alternative path 1; and if node 4 is also upgraded, then the ISP can also activate alternative path 2 , if needed.

To formulate this as an optimization problem, we introduce a set $\mathcal{P}_{f}$ for each flow $f$. This set includes alternative paths that flow $f$ can follow to reach its destination. For example, an ISP can analyze historical network data to predict which paths will be underutilized, and hence they can be dynamically activated to carry flow $f$ when its shortest path becomes congested. For each alternative path $p \in \mathcal{P}_{f}$, we define a group of nodes $s_{p f}$ which all need to be upgraded to SDN in order for path $p$ to become available to route flow $f$. For example, in Figure 1 we have $s_{\text {alternative_path } \_1, f}=\{1\}$ and $s_{\text {alternative_path_2 } 2, f}=\{1,4\}$. A generic approach to compute these groups was presented in [18], [19].

There will be a TE benefit $w_{p f t} \geq 0$ if alternative path $p \in \mathcal{P}_{f}$ is available for routing flow $f$ at time period $t$. A similar modeling approach of TE benefit has been considered in [18], [19]. This benefit will be higher for flows that carry large volumes of traffic and paths with sufficient spare capacity to carry these volumes. For a given upgrading policy $\boldsymbol{x}$, the total TE benefit (or flexibility) can be expressed as follows:

$$
J_{2}(\boldsymbol{x})=\sum_{t \in \mathcal{T}} \sum_{f \in \mathcal{F}} \sum_{p \in \mathcal{P}_{f}} w_{p f t} 1_{\left\{\prod_{n \in s_{p f}}\left[\sum_{t^{\prime} \leq t} x_{n t^{\prime}}\right]>0\right\}} .
$$

Here, the benefit $w_{p f t}$ is earned when all nodes in the group $s_{p f}$ have been upgraded to SDN by time $t$. In the special case that $w_{p f t}=1 \forall p, f, t$, the total TE benefit matches the total number of alternative paths that are enabled by SDN nodes.

The objective of the ISP is to find the SDN upgrading policy that maximizes the TE flexibility (TEFM problem):

$$
\begin{array}{ccc}
\text { Obj2 : } & \max _{\boldsymbol{x}} & J_{2}(\boldsymbol{x}) \\
& \text { s.t. } & \text { constraints }:(2),(3),(5)
\end{array}
$$

Obj2 is more complex than Obj1, since it depends on the exact set of SDN nodes that each flow traverses, rather than on whether the flow traverses at least one of them. In this section, we present an initial approach to optimize this objective. Our 
idea is to express the TEFM problem as the maximization of a set function with bounded supermodular degree [11]. We begin with the following definitions.

Definition 2: The supermodular degree of an element $g \in \mathcal{G}$ by a function $H($.$) is defined as the cardinality of the set$ $\mathcal{D}_{H}^{+}(g):=\left\{g^{\prime} \in \mathcal{G} \quad: \quad \exists \mathcal{X} \subseteq \mathcal{G}\right.$ for which $H\left(\mathcal{X} \cup\left\{g^{\prime}\right\} \cup\right.$ $\left.\{g\})-H\left(\mathcal{X} \cup\left\{g^{\prime}\right\}\right)>H(\mathcal{X} \cup\{g\})-H(\mathcal{X})\right\}$. In other words, the set $\mathcal{D}_{H}^{+}(g)$ contains all elements $g^{\prime}$ the existence of which in a set might increase the marginal value of element $g$.

Definition 3: The supermodular degree of a function $H($.$) ,$ denoted by $D_{H}^{+}$, is simply the maximum supermodular degree of any element $g \in \mathcal{G}$. Formally, $D_{H}^{+}=\max _{g \in \mathcal{G}}\left|\mathcal{D}_{H}^{+}(g)\right|$.

It is not hard to express $J_{2}$ as a function with bounded supermodular degree $\left(D_{J_{2}}^{+}\right)$. In fact, all the nodes that are included in the same group depend on each other, in the sense that upgrading only one of them yields no TE benefit, but when they are all upgraded a benefit is earned ( $w_{p f t}$ for group $s_{p f}$ ). For example, in Figure 1, the marginal value for upgrading node 4 is zero if node 1 is not already upgraded, since the only available path for the flow would be still the shortest path. But, if node 1 is already upgraded, then the marginal value for upgrading node 4 becomes positive (since routing over alternative path 2 now becomes possible). Hence, the supermodular degree of function $J_{2}$ is 1 in this example. For a single time period, the supermodular degree of $J_{2}$ is simply the maximum number of nodes that share a same group with any other node. In general, it holds $0 \leq D_{J_{2}}^{+} \leq N T$.

A variant of the greedy algorithm has been proposed for maximizing any function $H$ with bounded supermodular degree $D_{H}^{+}$. We call this the Super-greedy algorithm (see Algorithm 3). This algorithm starts with an empty solution set $\mathcal{X}$ (line 1 ) and iteratively augments subsets of elements to it (lines 2-4). At each iteration, it picks an element $g^{*}$ and a subset of those elements that increase the marginal value of $g^{*}$, i.e., a subset of the set $\mathcal{D}_{H}^{+}\left(g^{*}\right)$. The above choice is made greedily, so that the highest marginal benefit is earned. The procedure ends when there are no more elements to augment.

The work in [11] has shown that Super-greedy achieves an approximation ratio for the problem of maximizing a function with bounded supermodular degree. Nevertheless, a necessary condition for this result to hold is that the constraints of the problem form a k-extendible system, which is a class of constraints that captures the case of $k$ matroid constraints, but not the case of knapsack constraints. For the special case that all the upgrading costs are equal, the constraints (2), (3) can be expressed as $k=2$ matroid constraints. Therefore, we obtain:

Proposition 1: Super-greedy achieves an $\left(1 /\left(2\left(D_{J_{2}}^{+}+1\right)+\right.\right.$ 1))-approximation ratio to the TEFM problem for the special case of uniform upgrading costs.

\section{Dataset-DRiven Evaluation}

In this section, we evaluate the performance of the proposed algorithms using real-world network topologies and traffic matrices. Overall, we find that our approach can increase by $54 \%$ the amount of programmable traffic compared to stateof-the-art methods, especially in practical scenarios where the network is upgraded in a time window of four or five years. In general, the ISP acquires more benefits by spreading the upgrades over many instead of one year. Nevertheless,

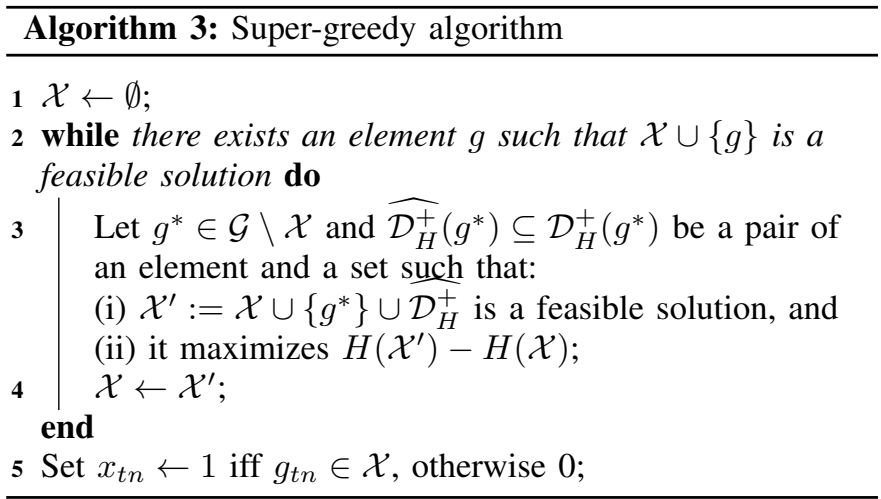

this strategy can be detrimental when the SDN costs are relatively stable over time (up to $20 \%$ drop per year). We also find that by optimizing the objective of programmable traffic maximization, benefits are also realized for the objective of TE flexibility maximization (and vise versa). However, there will be a performance loss (up to a factor of 2), since each algorithm favors one objective over the other.

We have implemented the following four algorithms:

1) DEG [20]: This scheme upgrades the nodes with the highest degrees (number of incoming and outgoing adjacent links) in the topology graph. All the upgrades take place at the first time period.

2) VOL [8], [20]: This scheme upgrades the nodes with the highest traffic volume that traverses them. All the upgrades take place at the first time period.

3) Modified-greedy: The proposed scheme in Algorithm 1 extended for many time periods.

4) Local search: The proposed scheme in Algorithm 2 for $\epsilon=2$ that can spread upgrades over many time periods.

The main part of the evaluation is carried out using the Abilene dataset [12] which is obtained from an educational backbone network in North America. This network consists of 12 nodes and 30 directed links. The dataset records the traffic matrix, i.e., the data transmitted between every pair of nodes, every 5 minutes for an overall period of six months. We use the traffic matrix at $8: 00 \mathrm{pm}$ in the first day to set the rates of the respective 144 flows. The aggregate rate is found to be $5.46 \mathrm{Gbps}$. These rates correspond to the $\lambda_{t f}$ values for period $t=1$. We increase the rates in subsequent periods (years) by $22 \%\left(\lambda_{t f}=\lambda_{t-1 f} \cdot 122 \%\right)$ [14]. The dataset also records the OSPF weights of all the links, which allows us to find the shortest path between every pair of nodes $\left(\mathcal{N}_{f}\right.$ sets).

We emphasize that we focus on this specific subset of the dataset because it represents a peak time period when SDN is more important. Moreover, this dataset is publicly available online, whereas data from most ISPs is proprietary. The evaluation code we wrote is publicly available online [21]. We believe that the reproducibility of the results will encourage future experimentation with SDN algorithms.

The first question we examine is how the proposed algorithms compare with the state-of-the-art methods. Since the latter neglect the timing issue, and in order to ensure a fair comparison, we begin our investigation with $T=1$ time period, i.e., all upgrades take place within one year. As a 


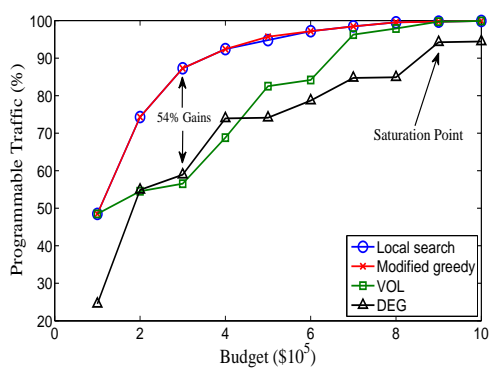

(a)

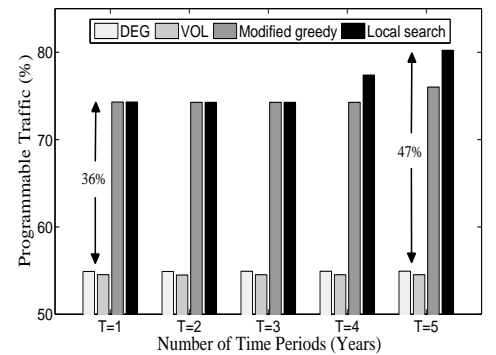

(b)

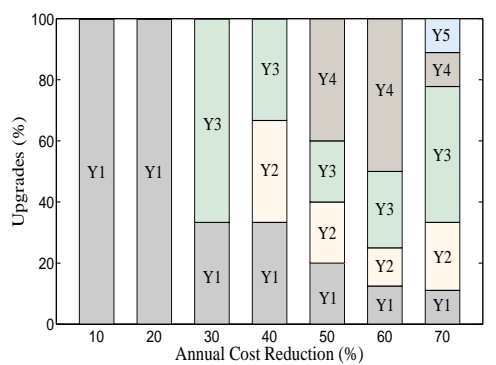

(c)

Fig. 3. The programmable traffic achieved by DEG, VOL, Modified greedy and Local search algorithms as a function of (a) the budget $B$ and (b) the number of time periods $T$. (c) The distribution of upgrades across years (Y1, Y2, Y3, Y4, Y5) for different cost reduction rates.
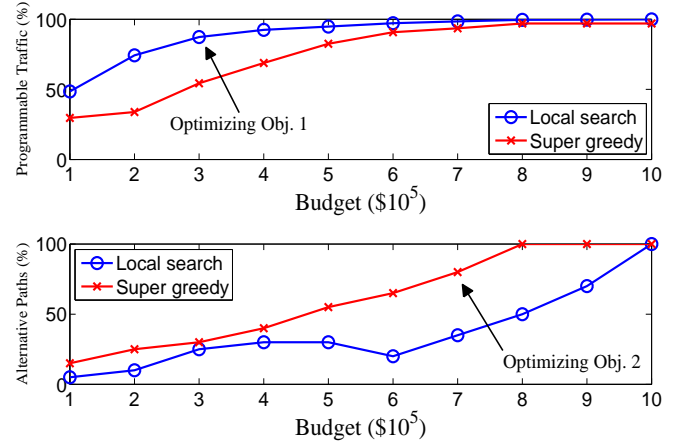

(a)

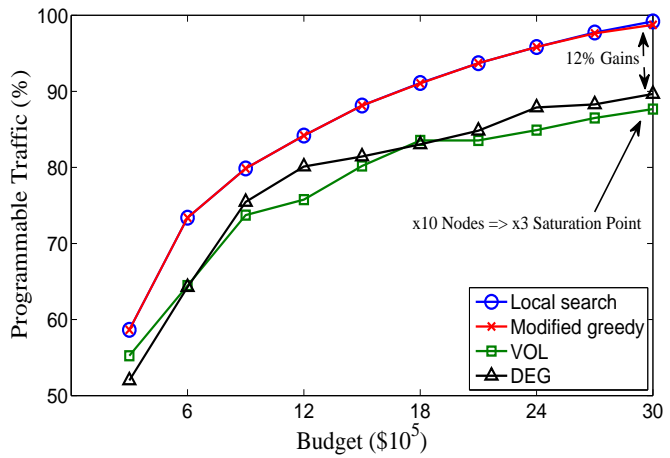

(b)

Fig. 4. (a) Impact of optimizing different objectives for $T=1$. (b) Programmable traffic for the Deltacom network [13].

canonical scenario, we set the cost of upgrading a node to SDN to $\$ 100 K$ [4] ( $b_{t n}$ values for $\left.t=1\right)$, and we vary the budget $B$ from $\$ 100 K$ to $\$ 1 M$ (Figure 3(a)). We observe that as the budget increases, the volume of programmable traffic increases for all algorithms. This is because more nodes are upgraded to SDN which creates more opportunities for the flows to traverse SDN nodes. There exists a saturation point $(B=\$ 900 K)$, after which no significant changes are noticed. The proposed algorithms (Modified greedy and Local search) achieve up to $54 \%$ more programmable traffic than their counterparts.

We then explore the impact of the number of time periods $T$ in Figure 3(b). Here, we keep $B=\$ 200 K$ constant, but we vary $T$ within 1 to 5 years. To capture technology maturity, we decrease the SDN upgrading costs by $40 \%$ per year, i.e., $b_{t n}=b_{t-1 n}-b_{t-1 n} \cdot 40 \%$. For $T=1$, the results match those in Figure 3(a). For $T>1$, additional benefits can be acquired by postponing some of the upgrades after the first year when the costs will be lower. Local search algorithm intelligently spreads the upgrades across different years to achieve the best performance among the four algorithms. The benefits over the state-of-the-art methods are up to $47 \%$, and $5.5 \%$ over Modified greedy for $T=5$.

In Figure 3(c), we take a closer look into the distribution of upgrades over years when the Local search algorithm is used. We evaluate various scenarios which differ into the annual decrease rate of the upgrading costs. We find that for relatively low rates of cost decrease (up to 20\%), all the upgrades should take place within the first year. But, after this point, it is more beneficial to postpone some of the upgrades in future. The distribution of upgrades over years becomes more diverse as the rate of cost decrease increases.

We also explore the interplay between traffic programmability and TE flexibility benefits. As we showed in previous figures, Local search is in practice a very efficient algorithm for maximizing programmable traffic. But, a large volume of programmable traffic cannot guarantee by itself a large number of alternative routing paths (and vise versa). Therefore, it is questionable how well an algorithm that optimizes one of the two objectives will perform with respect to the other objective. Figure 4(a) aims to shed light on this issue by comparing the performance of the Local search algorithm (which optimizes programmable traffic) and Super-greedy (which optimizes TE flexibility). Here, to model the TE benefits, we focus on the 10 flows with the highest rate, for which TE is most important. Then, we consider as alternative paths the second and third shortest path for each flow that do not overlap with the shortest path $\left(\mathcal{P}_{f}\right.$ sets). We find that by optimizing one of the objectives, benefits are realized also for the other objective. However, there will be a performance loss (up to a factor of 2), since each algorithm favors one objective over the other.

Although in our evaluation we used a real network topology and traffic matrices, it would be also interesting to study the results in larger networks. Towards this goal, we use the topology of the Deltacom backbone network in North America, which consists of 113 nodes and 161 links, and it is publicly available online in [13]. Since there is no available information about the traffic, we generate this artificially. Particularly, we create $F=1,000$ flows, by picking uniformly 
at random origin-destination pairs. We compute the shortest paths based on the hop count length, and we set the flow rate to be disproportional to it (following the gravity model [22]). In Figure 4(b), we repeat the experiment presented in Figure 3(a), but for this larger network. We find that the proposed algorithms perform up to $12 \%$ better than their counterparts. The saturation point is found to be $B=\$ 3 M$, about three times larger than in the small network. We attribute this difference to the larger number of nodes (10x) in the Deltacom network and the topological characteristics as the Deltacom has a higher link density which enables SDN nodes to cover more flows. The running times of the algorithms are typically on the scale of minutes for the small network and hours for the large network. These are acceptable running times in practice, since the problem has to be solved offline by the ISP.

\section{RELATED WORK}

Incremental deployment of new protocols and architectures is an operational paradigm shift [2], and SDN is no exception to that. Namely, several techno-economic factors make ISPs reluctant to proceed with immediate full-scale SDN deployment. This renders hybrid SDN networks an imperative intermediate step [3]. Such systems are nowadays possible due to hybrid routers [23], yet their deployment is not without challenges. For example, the co-existence of multiple control planes poses risks for fault-free routing, and specific measures should be taken to avoid this, e.g., see [24].

One of the key traffic engineering goals in these hybrid networks is to use the SDN routers so as to minimize the load of congested links, e.g., see seminal work [6], and [20], [25]. For a given set of upgraded nodes, this is an LP problem. However, upgrading decisions are more challenging as they yield intractable problem formulations. Reference [20] proposed meaningful heuristic algorithms and evaluated them using actual network data. Our objective is different, i.e., we maximize the amount of programmable traffic similarly to [8], [9], and we additionally increase the TE flexibility. This latter property enables dynamic responses to link failures and to temporal link congestion, as alternative paths can be activated on demand. Therefore, the resulting architecture is robust to uncertainties about future network state.

Besides, one of our main focal points is the impact of upgrade timing. This is a very crucial and practical issue in hybrid SDNs given that (i) new technology costs reduce rapidly [26], (ii) the out-of-phase life-cycles of the legacy devices render cost-prohibitive massive replacements, and (iii) the practical, technical and security limitations render impossible one-time upgrades. Prior works as those above or others, e.g., [27], do not focus on these aspects. On the contrary, few prior interesting works [18], [19] studied gradual upgrades, yet, they do not provide tight bounds, nor they analyze the impact of equipment cost reduction.

Prior work also tries to achieve SDN-like flexible path enforcement with legacy networks. Fibbing [28] injects fake nodes and links into the underlying link state routing protocol to achieve some level of load balancing and TE, but its forwarding rule matching is limited to destination-based and its expressivity is thus confined to expressivity of IP routing.

\section{CONCLUSION}

In this paper, we studied the migration to SDN of highend cost ISP networks. To this end, we introduced a model of SDN upgrades general enough to capture different migration costs, as well as two plausible ISP objectives. An ISP can apply our methodology to optimally decide which nodes to upgrade over a period that may span several years. Using two real-world network topologies and traffic matrices, we differentiated situations in which upgrades should be spread over many instead of one step, and explored the interplay between the different objectives.

\section{REFERENCES}

[1] W. Xia, Y. Wen, C. Foh, D. Niyato, "A Survey on Software-defined Networking", IEEE Commun. Surveys \& Tutor, vol. 17, no. 1, 2015.

[2] M. K. Mukerjee, D. Han, S. Seshan, P. Steenkiste, "Understanding Tradeoffs in Incremental Deployment of New Network Architectures", in Proc. of ACM CoNEXT, 2013.

[3] S. Vissicchio, L. Vanberer, O. Bonaventure, "Opportunities and Research Challenges of Hybrid Software Defined Networks", ACM CCR, vol. 44, no. 2, 2014.

[4] Reuters Technology News, "AT\&T to buy Cisco Core Routers for Network Upgrade", [Online:] www.reuters.com

[5] Z. Cao, M. Kodialam, T. V. Lakshman, "Traffic Steering in Software Defined Networks: Planning and Online Routing", ACM DCC, 2014.

[6] S. Agarwal, M. Kodialam, T.V. Lakshman, "Traffic Engineering in Software Defined Networks", IEEE Infocom, 2013.

[7] Light Reading Portal, "NEC Slashes OpenFlow SDN Controller Pricing", [Online]: http://www.lightreading.com/carrier-sdn/sdn-technology/ nec-slashes-openflow-sdn-controller-pricing/d/d-id/711391, 2014.

[8] D. Levin, M. Canini, S. Schmid, F. Schaffert, A. Feldmann, "Panopticon: Reaping the Benefits of Incremental SDN Deployment in Enterprise Networks", in Proc. of USENIX ATC, 2014.

[9] T. Lukovszki, M. Rost, S. Schmid,"It's a Match! Near-Optimal and Incremental Middlebox Deployment", $A C M C C R$, vol. 46, no. 1, 2016.

[10] J. Lee, M. Sviridenko, J. Vondrak, "Submodular Maximization over Multiple Matroids via Generalized Exchange Properties", Math. of Operations Research, vol. 35, no. 4, 2010.

[11] M. Feldman, R. Izsak, "Constrained Monotone Function Maximization and the Supermodular Degree", in Proc. of APPROX/RANDOM, 2014.

[12] Abilene dataset, http://www.cs.utexas.edu/ yzhang/research/AbileneTM

[13] "The Internet Topology Zoo", http://www.topology-zoo.org/dataset.html

[14] Cisco White Paper, "VNI: Forecast and Methodology, 2015-2020", 2016.

[15] S. Khuller, A. Moss, J. Naor, "The Budgeted Coverage Problem", Information Processing Letters, vol. 70, no. 1, 1999.

[16] C. Chekuri, J. Vondrák, R. Zenklusen, "Randomized Pipage Rounding for Matroid Polytopes and Applications", in Proc. of FOCS, 2010.

[17] A. Gupta, V. Nagarajan, R. Ravi, "Robust and MaxMin Optimization under Matroid and Knapsack Uncertainty Sets", ACM Transactions on Algorithms, vol. 12, no. 1, 2016.

[18] M. Caria, A. Jukan, M. Hoffmann,"A Performance Study of Network Migration to SDN-enabled Traffic Engineering”, IEEE Globecom, 2013.

[19] T. Das, M. Caria, A. Jukan, M. Hoffmann, "A Techno-economic Analysis of Network Migration to Software-Defined Networking", arXiv. 1310.0216v1, 2013.

[20] D.K. Hong, Y. Ma, S. Banerjee, Z.M. Mao, "Incremental Deployment of SDN in Hybrid Enterprise and ISP Networks", ACM SOSR, 2016.

[21] Publicly available code: goo.gl/EXoZZZ

[22] P. Tune, M. Roughan, "Internet Traffic Matrices: A Primer", Recent Advances in Networking, vol. 1, 2013.

[23] ONF, “OpenFlow Switch Specification”, March 2015.

[24] S. Vissicchio, L. Cittadini, O. Bonaventure, G. G. Xie, L. Vanberer, "On the Co-Existence of Distributed and Centralized Routing Control-Planes", in Proc. of IEEE Infocom, 2015.

[25] Y. Guo, Z. Wang, X. Yin, X. Shi, J. Wu, "Traffic Engineering in SDN/OSPF Hybrid Network", in Proc. of IEEE ICNP, 2014.

[26] L. Duan, J. Huang, J. C. Walrand, "Economic Analysis of 4G Upgrade Timing", IEEE Trans. Mob. Comput, vol. 14, no. 5, 2015.

[27] C.-Y. Chu, K. Xi, M. Luo, H. Chao, "Congestion-aware Single Link Failure Recovery in Hybrid SDN Networks", IEEE Infocom, 2015.

[28] S. Vissicchio, O. Tilmans, L. Vanbever, and J. Rexford, "Central Control over Distributed Routing”, in Prof. of ACM SIGCOMM, 2015. 